\title{
Statistical Modeling of Plume Exhausted from Herschel Small Nozzle with Baffle
}

\author{
Gennady Markelov ${ }^{1}$ and Juergen Kroeker ${ }^{2}$ \\ 1 AOES Group BV, Pustbus 342, 2300 AH Leiden, The Netherlands \\ Gennady.Markelov@aoes.com \\ ${ }^{2}$ EADS Astrium GmbH, Friedrichshafen, Germany
}

\begin{abstract}
Constantly released Helium on board of the Herschel spacecraft is used to cool three scientific instruments down to $0.3 \mathrm{~K}$. The Helium is released by small nozzles creating a counter-torque. This compensates the torque caused by the solar pressure acting on the spacecraft surfaces. An optimization of the nozzle shape did not allow avoiding severe plume impingement on the spacecraft surfaces and consequently the application of baffles has been considered to reduce plume impingement effects. Two baffle shapes, cylindrical and conical, with different radii and lengths have been analyzed numerically. The analysis has been performed with a kinetic approach, namely, the direct simulation Monte Carlo (DSMC) method to cope with changing flow regime from continuum in a subsonic part of the nozzle through transitional to freemolecular flow inside the baffle. A direct application of DSMC-based software would require large computer resources to model the nozzle and plume flows simultaneously. Therefore, the computation was split in two successive computations for the nozzle and nozzle/plume flow. Computations of plume flow with and without baffles were performed to study effects of baffle size and shape on the plume divergence, and plume impingement on the Herschel spacecraft. It has been shown that small baffles even widen the plume. An increase of radius/length of the baffle decreases the plume divergence, however even the largest baffle could not meet the requirements.
\end{abstract}

\section{Introduction}

The 'Herschel Space Observatory' is part of the fourth cornerstone mission in the 'Horizons 2000' program of the European Space Agency (ESA), with the objectives to study the formation of galaxies in the early universe and the creation of stars. In a dual launch together with Planck, Herschel will be placed in an operational Lissajous orbit around the Earth-Sun L2 point by an Ariane 5 in 2008 to perform photometer and spectrometer measurements, covering the full far infrared to sub-millimetre wavelength range from 60 to 670 micrometers during its operational lifetime of 3.5 years. The prime contractor for Herschel/Planck is ThalesAlenia Space in Cannes, France, while the Herschel Payload Module is developed, built and tested under responsibility of EADS Astrium Spacecrafts in Friedrichshafen, Germany. 
The released Helium creates at the nozzles a counter-torque, which is used to compensate the torque caused by the solar pressure acting on the spacecraft. This counter-torque is partly neutralized by the Helium plume impingement on the spacecraft surfaces. To reduce the effect of the plume impingement, $95 \%$ of the total thrust has to be within a half-cone of $30 \mathrm{deg}$ in a distance of $0.5 \mathrm{~m}$ from the nozzle. To achieve the goal the following investigations have been performed:

- Optimization of the nozzle geometry to decrease a plume divergence,

- Application of a baffle for further decrease of the divergence (the baffle shall be small and have a simple shape),

- Definition of proper cant angle and nozzle locations if the design goal could not be achieved by the above activities.

Plume exhausted from a nozzle in a hard vacuum is characterized by the presence of all flow regimes, from continuum in the nozzle and even in the plume near field through transitional to free-molecular flow at a large distance from the nozzle. Modelling of such flows requires a special approach, for example, a successive application of continuum and kinetic methods (see [1] and refs. herein). The given problem is complicated by the facts that 1) a transitional flow regime occurs inside the nozzle due to a small mass flow rate and 2) a baffle can affect the flow at the nozzle exit plane and, probably, even inside the supersonic part of the nozzle. This complicates the splitting of the computational domain into regions and the application of proper numerical methods modelling the flow inside the domains.

This paper applies the direct simulation Monte Carlo (DSMC) method [2] to model plume flow with and without baffles, study effects of baffle size and shape on the plume divergence, and plume impingement on the Herschel spacecraft. This method is a computer simulation of movement and collisions of particles and it applies a statistical approach to perform the collisions. This is the most widely used numerical method in the area of rarefied gas dynamics and it was successfully applied to model plume flows and near continuum flows.

\section{Nozzle and Baffle Geometries}

Initially, the nozzle had the supersonic part with an half-angle of $15 \mathrm{deg}$ and exit diameter of $5.45 \mathrm{~mm}$. Temperature of helium at the nozzle inlet is $69 \mathrm{~K}$ and mass flow rate is $1.1 \mathrm{mg} / \mathrm{sec}$. The nozzle creates a rather wide plume with about $66 \%$ of total thrust within a half-cone of $30 \mathrm{deg}$. An optimization of the nozzle shape allowed to increase a plume collimation and achieve a plume shape with $74 \%$ of total thrust inside a half-cone of $30 \mathrm{deg}$. The optimal nozzle has a larger supersonic part: the half-cone angle of $32 \mathrm{deg}$ and exit diameter of 15 mm (Fig. 1 left). However, this nozzle does not meet the design goal, 95\%, and plume still impinges on the Herschel spacecraft surface. Figure 1 right shows the surface distribution of torque created by the plumes. The plumes impinge mainly the SVM shield, spacecraft body and the radiators creating a torque acting on the spacecraft body. To decrease further the plume divergence, the 

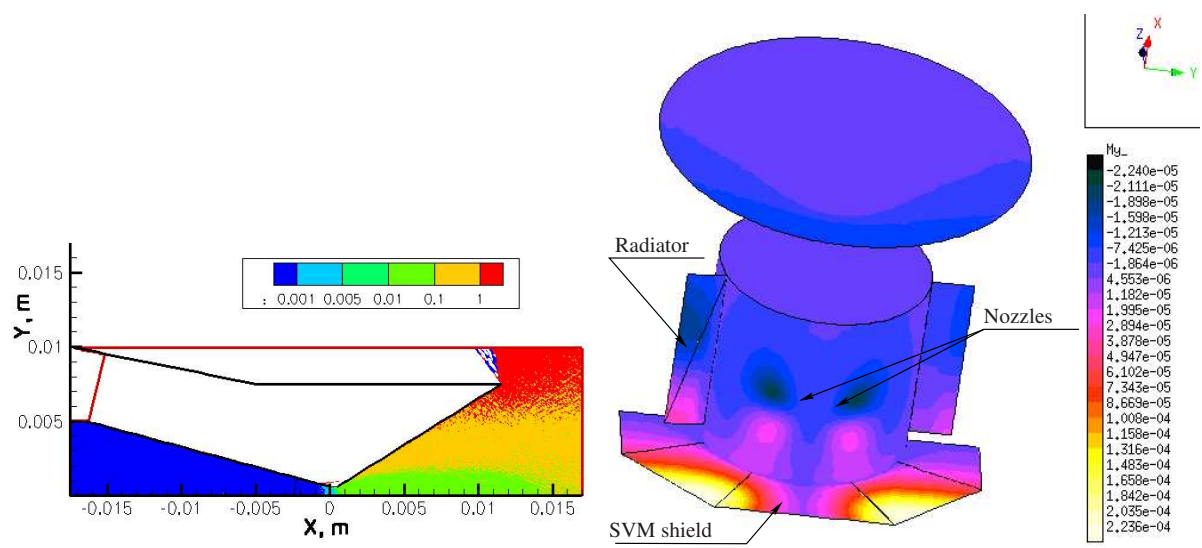

Fig. 1. Local Knudsen number flow-field for the optimal nozzle (left) and My torque distribution over the spacecraft surface (nozzles without baffles, values in $N / m$ )
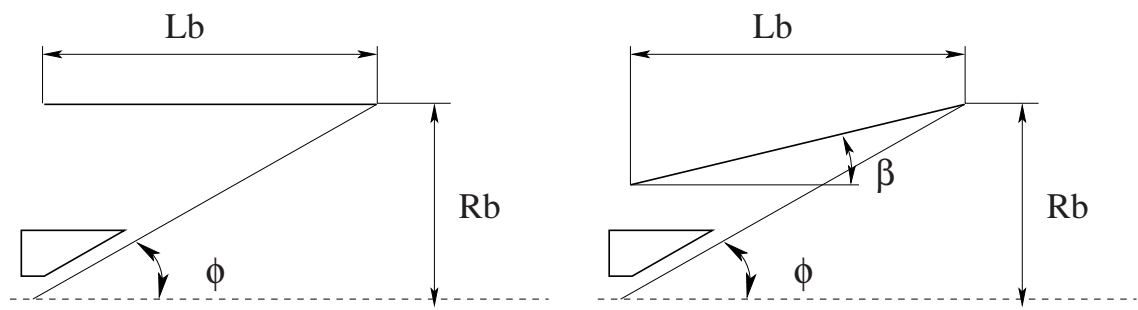

Fig. 2. Cylindrical (left) and conical (right) baffles

application of baffles has been considered. The baffles shall have a simple shape, either cylindrical or conical. Figure 2 shows geometrical parameters of the baffle where $L_{b}$ is an axial length, $R_{b}$ is a radius, and $\beta$ is the baffle angle.

\section{Numerical Approach}

Modeling of plume flow is quite complex from a numerical view point because as it includes different flow regimes, a continuum regime in the nozzle and transitional and free-molecular in the far plume field. For the Herschel small nozzle the transitional regime occurs already inside the nozzle due to the small mass flow rate. For example, values of local Knudsen number are larger then a threshold value 0.1 , which defines a border between continuum and transitional regimes (see Fig. 1left). Therefore, the kinetic approach has to be applied inside the nozzle already. Computations were performed with DSMC-based software, SMILE [3]. The software has 20 year history of development and is validated mutually. A variable hard sphere model [2] was applied to model intermolecular 
collisions and diffuse reflection with complete energy accommodation was used as a gas/surface interaction model.

To perform collisions between model particles, SMILE uses the Cartesian uniform grid. Each cell of the grid can be subdivided into smaller Cartesian cells to meet the method requirements on a linear size of the collisional cell. This allows implementation of an efficient algorithm to trace the model particles. The uniform cells are used as a base for other algorithms: radial weights and parallel algorithms. The radial weight is assigned for each cells strip along $\mathrm{X}$-axis to control a number of the model particles and make their distribution more uniform in radial direction. A parallel algorithm applies a static distribution of these cells between processors and cells are distributed to the processors on statistical base. In this case all processors communicate with each other. However, this algorithm allows a good load balance for a small number of processors and, as a result, an efficient use of the parallel computer [4. It is desirable to have the cells small enough to make these algorithms efficient.

The plume has to be computed up to a distance of 0.5 meters from the nozzle and collisional cells have to be small inside the nozzle and large in the plume far field. By adaptation of the uniform cells (subdivision into smaller collisional cells) the flow resolution in any place of computational domain can be achieved. However, even for a grid 1000x1000 cells in axial and radial directions, respectively, the nozzle locates inside two/three cells. This leads to very inefficient use of the software due to large load imbalance over processors and requires large computer resources. To reduce requirements to computer resources, a computation of nozzle and plume flows has been split into the following two successive computations:

1. modeling of flow inside the nozzle and in the vicinity of the nozzle exit,

2. modeling of the nozzle flow from the nozzle throat and plume flow.

The two-step approach allows us to reduce the requirements, significantly. An additional benefit is that the first computation has been done only once for all the baffle geometries and it requires more computer resources then the second computation. Numerical solutions inside the supersonic part of the nozzle are exactly the same for both computations. In principal, the second computation could be started using an inflow boundary located downstream from the nozzle throat. In this case, a velocity distribution function has to be sampled along this boundary. Otherwise, any simplification of the function, for example, application of ellipsoidal Maxwellian decreases the solution accuracy.

For the first computation an efficiency of the computational cluster used is not less then $85 \%$ using up to 28 processors. However, the second computation is very inefficient as the nozzle flow occupies a few cells only, which yields a poor load balancing. For example, an increase of number of processors from 2 to 8 yields a growth of wall clock time required to perform the computation. An improvement has been achieved by redistributing the cells over processors. For the redistribution, the computation has been stopped at certain moments and the work load has been estimated for each cell by comparing the time spent 
by each processor and number of model particles in that cell. Then cells were grouped along Y-axis to have an approximately equal work load over the groups. This redistribution also reduces the communication between processors as each processor has now a closed sub-domain and not loose cells in the entire computational domain. After this cell redistribution the efficiency has been increased up to $80 \%$ using four processors.

\section{$4 \quad$ Numerical Results}

Computations of plume flow have been performed for the optimal nozzle geometry. This section uses the following parameters to describe plume properties at a distance of $0.5 \mathrm{~m}$ from the nozzle. $t_{30}$ is the fraction of total thrust, $t$, within a half-angle of $30 \mathrm{deg}, \alpha_{95}$ is a half-angle, which includes $95 \%$ of total thrust. $\dot{m}$ and $t_{a}$ are mass flow rate and thrust along a plume centerline.

\subsection{Cylindrical Tube}

A geometry of the cylindrical tube is defined by two parameters, length and radius. Computations have been performed to study effects of both parameters on the plume divergence and the obtained results are shown in this section.

Effect of the tube length. The cylindrical tubes with a length of $L_{b}=63,100$, and $250 \mathrm{~mm}$ have been considered. The tube diameter is calculated assuming that the tube trailing edge is defined by a half-cone angle of $\phi=30 \mathrm{deg}$. A tip of the cone is near the beginning of the throat.

Figures 3 and 4 show Mach number flow fields for all these tubes. Helium atoms reflect on the tube surface in accordance with diffuse reflection. Some of Helium atoms go back to the nozzle and disturb the plume near field flow. An application of the tube makes the plume more collimated in terms of $t_{30}$ (Table1). However, tubes with 63-100 mm length create a more divergent plume in terms of $\alpha_{95}$ parameter. Only an application of $250 \mathrm{~mm}$ tube leads to very collimated plume with $t_{30}=0.901$, which is close to the design goal of the Herschel small nozzles.

The influence of the tube on the plume structure is clearly seen using a density distribution at a distance of $0.5 \mathrm{~m}$ from the nozzle (Fig. 4 right). The tube creates a sudden drop in the density distribution at $30 \mathrm{deg}$ half-cone angle and this drop is larger and sharper for larger tube lengths. Tubes with a length of $63-100 \mathrm{~mm}$ create lower density at angles less than $15 \mathrm{deg}$. The tube with the length of 250 mm provides higher density for angles up to 30 deg and lower density at larger angles comparing with the plume created by the bare nozzle.

Due to an open left hand end of the tube, $0.1 \mathrm{mg} / \mathrm{sec}$ Helium is released in the opposite direction for the $250 \mathrm{~mm}$ tube. When the left hand end is closed, Helium flows only along $\mathrm{X}$ direction. However, the closed end causes a slight plume divergence, for example, $100 \mathrm{~mm}$ tube creates a wider plume as the open tube with $63 \mathrm{~mm}$ length (cf. Tables 1 and 2). 

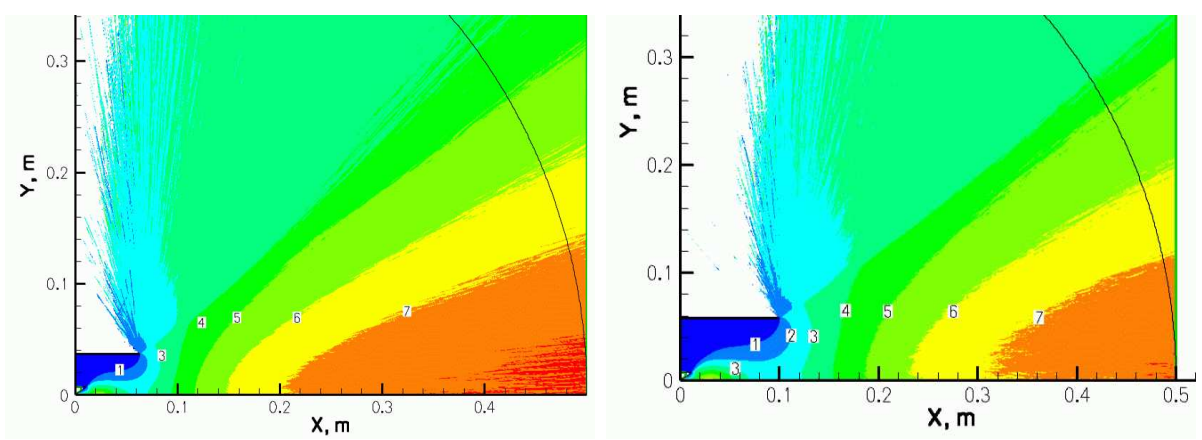

Fig. 3. Mach number flow field for tubes with a length of $63 \mathrm{~mm}$ (left) and $100 \mathrm{~mm}$ (right)
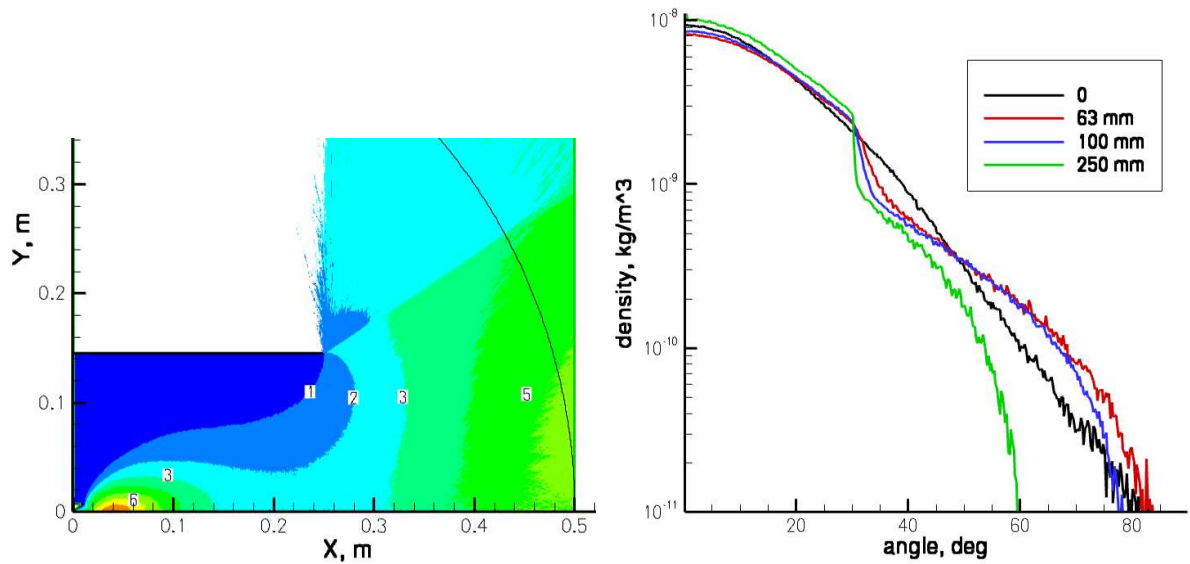

Fig. 4. Mach number flow field for a tube with a length of $250 \mathrm{~mm}$ (left) and density distribution at a distance of $0.5 \mathrm{~m}$ (right)

Effect of the tube radius. The effect has been investigated for tubes with a length of $100 \mathrm{~mm}$ and $250 \mathrm{~mm}$. The radius of $100 \mathrm{~mm}$ tube was decreased from $58.34 \mathrm{~mm}$ down to $40 \mathrm{~mm}$. As a result, the trailing edge of the baffle is on the half-cone angle of $\phi=20 \mathrm{deg}$. From an intuitive view-point, this should increase the plume collimation. However, a small baffle leads to a large disturbance of the flow in the near plume field where intermolecular collisions occur. As a result,

Table 1. The plume properties for tubes

\begin{tabular}{lccccc}
\hline length, $\mathrm{mm}$ & $t_{30}$ & \multicolumn{2}{c}{$\alpha_{95}, \mathrm{deg} \dot{m}, \mathrm{mg} / \mathrm{sec}$} & $t_{a}, \mathrm{mN}$ & $t, \mathrm{mN}$ \\
\hline 0 & 0.741 & 46.64 & 1.10 & 0.833 & 0.925 \\
63 & 0.763 & 51.98 & 1.02 & 0.760 & 0.851 \\
100 & 0.799 & 50.31 & 1.01 & 0.751 & 0.834 \\
250 & 0.901 & 37.23 & 0.99 & 0.748 & 0.808 \\
\hline
\end{tabular}


Table 2. The plume properties for tubes with closed left end

\begin{tabular}{lccccc}
\hline length, $\mathrm{mm}$ & $t_{30}$ & \multicolumn{2}{c}{$\alpha_{95}, \mathrm{deg}$} & $\dot{m}, \mathrm{mg} / \mathrm{sec}$ & $t_{a}, \mathrm{mN} t, \mathrm{mN}$ \\
\hline 100 & 0.763 & 51.76 & 1.09 & 0.801 & 0.898 \\
250 & 0.877 & 38.86 & 1.09 & 0.807 & 0.877 \\
\hline
\end{tabular}

Table 3. Effect of tube radius on plume properties

\begin{tabular}{lccccccc}
\hline \multicolumn{2}{l}{$L_{b}, \mathrm{~mm}$} & $R_{b}, \mathrm{~mm}$ & $\phi, \mathrm{deg}$ & $t_{30}$ & \multicolumn{4}{c}{$\alpha_{95}, \mathrm{deg} \dot{m}, \mathrm{mg} / \mathrm{sec}$} & $t_{a}, \mathrm{mN}$ & $t, \mathrm{mN}$ \\
\hline 100 & 58.34 & 30 & 0.799 & 50.31 & 1.01 & 0.751 & 0.834 \\
100 & 40.00 & 20 & 0.637 & 56.43 & 0.92 & 0.623 & 0.726 \\
\hline 250 & 144.94 & 30 & 0.901 & 37.23 & 0.99 & 0.748 & 0.808 \\
250 & 91.59 & 20 & 0.832 & 41.30 & 0.86 & 0.612 & 0.674 \\
\hline
\end{tabular}

the tube with a radius of $40 \mathrm{~mm}$ creates a plume wider than the bare nozzle does (Table 3). Figure 5 shows that the density distribution for this plume does not have the significant drop at $20 \mathrm{deg}$ and the density in the core flow is lower then the correspondent values for larger tube radius.

A decrease of the tube radius for $250 \mathrm{~mm}$ length yields also a wider plume. In this case the baffle surface is still far from the nozzle (cf. Figs. 4 left and 5 right) and the plume is more collimated than without the baffle. The decrease of the tube radius does not affect the density distribution for small angles. However, the density drop is not big as it is for larger tube radius and, as a result, density is higher at angles larger than $30 \mathrm{deg}$.

\subsection{Conical Baffle}

Various conical baffles have been considered with half-cone angles of 5, 10, 15, and $20 \mathrm{deg}$. The length of the baffles is set to $L_{b}=100 \mathrm{~mm}$. The trailing edge
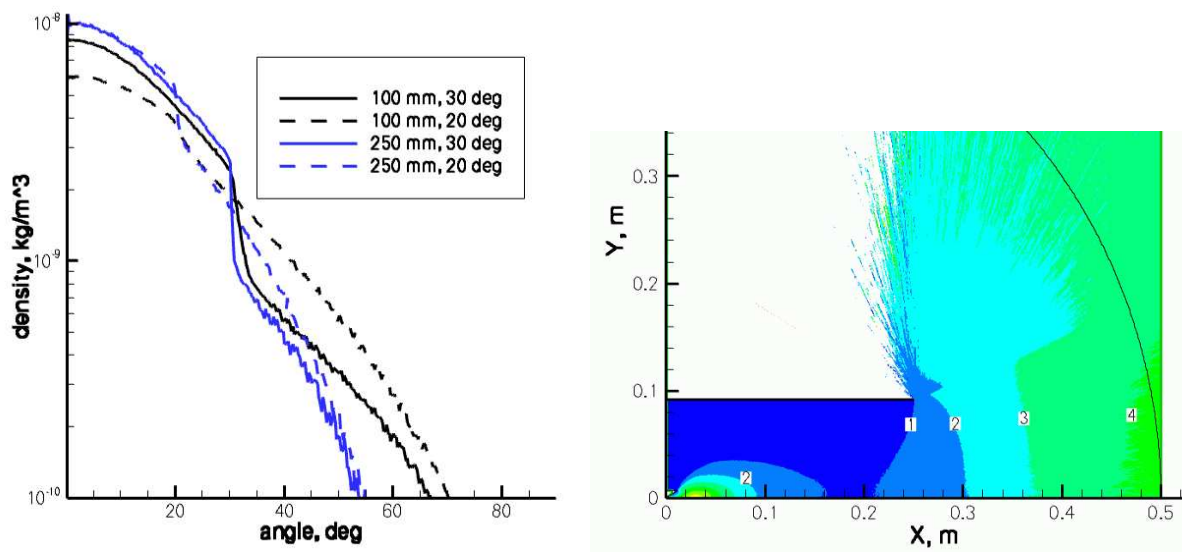

Fig. 5. Density distribution at a distance of $0.5 \mathrm{~m}$ for tubes (left) and Mach number flow field for a tube with a length of $250 \mathrm{~mm}$ and smaller radius (right) 

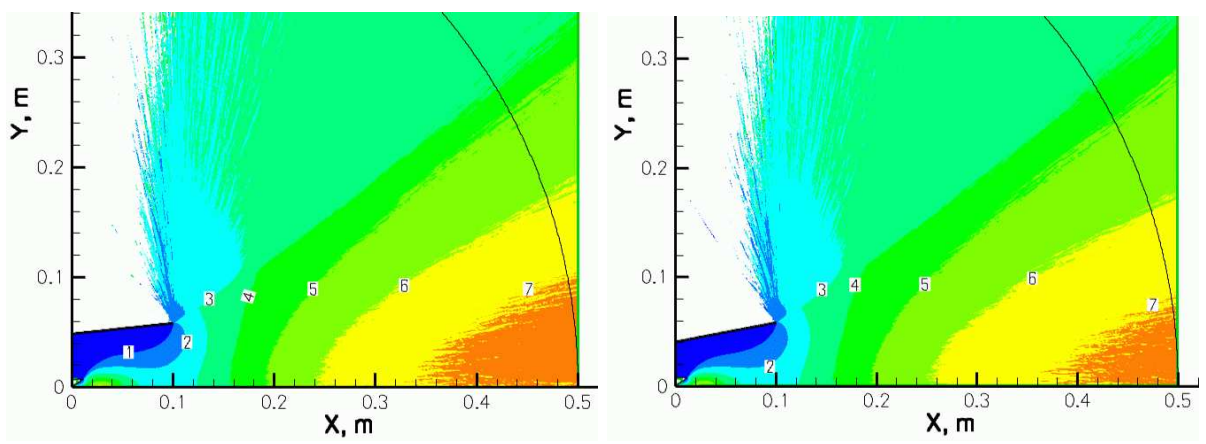

Fig. 6. Mach number flow field for a conical baffle with a length of $100 \mathrm{~mm}$ (left, 5 deg; right, $10 \mathrm{deg})$
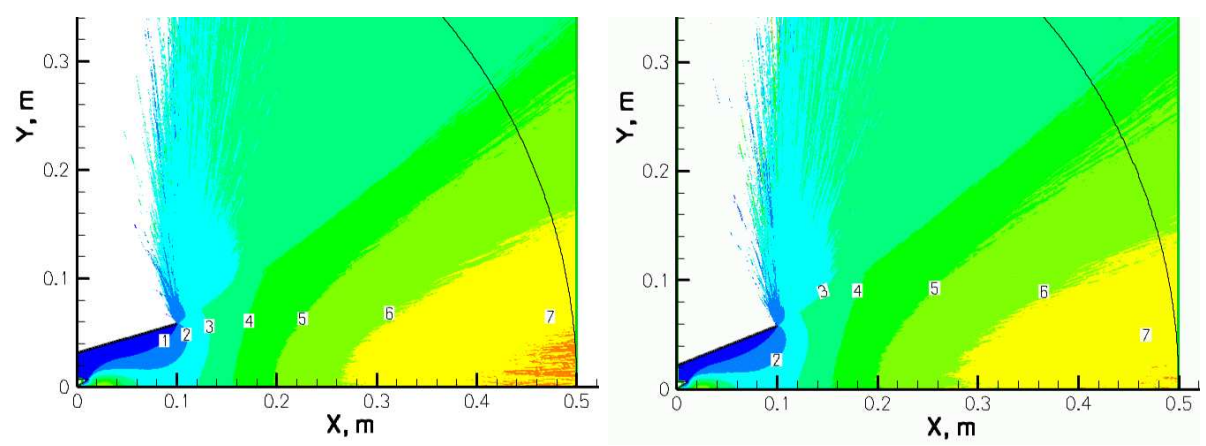

Fig. 7. Mach number flow field for a conical baffle with a length of $100 \mathrm{~mm}$ (left, 15 deg; right, $20 \mathrm{deg})$

position is defined by a half-cone angle of $30 \mathrm{deg}$. Figures [6 and 7 show Mach number flow-fields for these baffles. An increase of the baffle angle decreases very slightly the plume collimation in terms of $t_{30}$ (Table 4). The plausible reason is that the larger portion of Helium flux is emitted along X direction. At the same time the increase of $\beta$ leads to more collimated plume in terms of $\alpha_{95}$.

\subsection{Conclusions}

The Herschel spacecraft uses Helium to cool scientific instruments and to compensate the torque caused by the solar pressure acting on the spacecraft surface. The Helium is emitted through small nozzles and their design has to provide a minimum plume impingement. The flow in the nozzles and in the plume passes from continuum regime through transitional to free-molecular regime and only kinetic approach could handle such flows. The kinetic approach, namely, the direct simulation Monte Carlo method has been applied to perform a numerical analysis. 
Table 4. Cone angle effect on plume properties

\begin{tabular}{lccccc}
\hline$\beta, \operatorname{deg}$ & $t_{30}$ & $\alpha_{95}, \mathrm{deg}$ & $\dot{m}, \mathrm{mg} / \mathrm{sec}$ & $t_{a}, \mathrm{mN}$ & $t, \mathrm{mN}$ \\
\hline 0 & 0.799 & 50.31 & 1.01 & 0.751 & 0.834 \\
5 & 0.791 & 49.97 & 1.03 & 0.766 & 0.851 \\
10 & 0.786 & 49.83 & 1.05 & 0.778 & 0.865 \\
15 & 0.783 & 49.04 & 1.07 & 0.788 & 0.876 \\
20 & 0.779 & 47.88 & 1.08 & 0.800 & 0.888 \\
\hline
\end{tabular}

A straightforward application of DSMC-based software, SMILE, would have required enormous computer resources to model the nozzle and plume flows with the required accuracy. Consequently the nozzle and plume flow analyses have been split into two successive computations. In the first analysis the flow inside entire nozzle has been computed and then subsequent a computation of a flow in the supersonic part of nozzle and plume flow has been performed. The second computation has used as boundary conditions the results of the first analysis. This allowed us to reduce significantly requirements to computer resources and achieved the required accuracy.

To decrease the plume divergence and, hence, plume impingement on the spacecraft surface, the application of various baffle shapes, cylinders and cones, were investigated. It was shown that the small baffles created even a wider plume than a bare nozzle. An increase of radius/length of the cylindrical baffle decreases the plume divergence. The baffle with largest length and radius showed the best performance, close to the requirement. The application of the conical baffle increase the plume collimation, but no significant effect of the half-cone angle was observed.

\section{References}

1. Markelov, G.N.: Plume Impingement Analysis for Aeolus Spacecraft and Gas/Surface Interaction Models. J. Spacecraft Rockets 3, 607-618 (2007)

2. Bird, G.A.: Molecular Gas Dynamics and the Direct Simulation of Gas Flows. Pergamon Press, Oxford (1994)

3. Ivanov, M.S., Markelov, G.N., Gimelshein, S.F.: Statistical Simulation of Reactive Rarefied Flows: Numerical Approach and Applications. AIAA Paper 98-2669 (1998)

4. Ivanov, M., Markelov, G., Taylor, S., Watts, J.: Parallel DSMC strategies for 3D computations. In: Schiano, P., Ecer, A., Periaux, J., Satofuka, N. (eds.) Parallel CFD 1996, pp. 485-492. North Holland, Amsterdam (1997) 